\title{
B Acute Lymphoblastic Leukemia
}

National Cancer Institute

\section{Source}

National Cancer Institute. B Acute Lymphoblastic Leukemia. NCI Thesaurus. Code C8644.

The most frequent type of acute lymphoblastic leukemia. Approximately $75 \%$ of cases occur in children under six years of age. This is a good prognosis leukemia. In the pediatric age group the complete remission rate is approximately $95 \%$ and the disease free survival rate is $70 \%$. Approximately $80 \%$ of children appear to be cured. In the adult age group the complete remission rate is $60-85 \%$. (WHO, 2001) 